\title{
Hydrothermal Synthesis of Carbon Nanotubes-Polyaniline (CNT-PANI) Composites and Preliminary Electrochemical Characterization of CNT-PANI Coatings
}

\author{
LAURA-MADALINA CURSARU*, ANA-MARIA MOCIOIU, IOAN ALBERT TUDOR, \\ ROXANA MIOARA PITICESCU
}

National Research and Development Institute for Non-ferrous and Rare Metals, 102 Biruintei Blvd., 077145, Pantelimon, Ilfov, Romania

\begin{abstract}
Heavy metals have a major contribution to biosphere pollution due to toxicity. The detection and monitoring of the environmental agents in soil, water and air is very important for the general health of humans and animals. It has been recently shown that electrochemical techniques such as differential pulse voltammetry (DPV) and square wave anodic stripping voltammetry (SWASV) using modified electrodes are very attractive methods for detecting heavy metals. The aim of this paper is to demonstrate the potential of hydrothermal process combined with electrochemical techniques to obtain modified electrodes based on functionalized carbon nanotubes (CNTs) and polyaniline (PANI) for metals detection. Commercial multi-walled carbon nanotubes (MWCNT) were functionalized by a mixture of $\mathrm{HNO}_{3} / \mathrm{H}_{2} \mathrm{SO}_{4}$ and further used for hydrothermal synthesis of CNT-PANI composites with different mass ratios. The resulted powders were analyzed by spectral (Fourier-Transform Infrared Spectroscopy) and thermal (Differential Scanning Calorimetry) methods, and then dispersed in a surfactant/electrolyte solution for preliminary electrochemical experiments (cyclic voltammetry, $C V$ and DPV) to obtain modified electrodes. The influence of the CNT: PANI mass ratio and the synthesis time on the formation of composites with the desired structural and electrochemical properties were studied. It was found that CNT-PANI composite powder having mass ratio 1:4 and synthesis time $3 \mathrm{~h}$ has the best structural and thermal characteristics and formed a weakly conductive film on the surface of the glassy carbon electrode. Preliminary electrochemical tests revealed the electroactive forms of polyaniline, through the presence of characteristic oxidation peaks but also reduction peaks, corresponding to reversible redox reactions, demonstrating that glassy carbon electrode has been electrochemically modified with CNTPANI coatings. Further studies will be conducted to test the potential application of glassy carbon electrode modified with CNT-PANI coatings as electrochemical sensor for heavy metals detection.
\end{abstract}

Keywords: carbon nanotubes, polyaniline, hydrothermal synthesis, modified glassy carbon electrodes

\section{Introduction}

Heavy metals have a major contribution to biosphere pollution due to toxicity. The detection and monitoring of the environmental agents in soil, water and air is very important for the general health of humans and animals. Current methods for detecting metals that include spectral methods such as atomic absorption spectroscopy (AAS), inductively coupled plasma optical emission spectrometry (ICP-OES) and inductively coupled plasma mass spectrometry (ICP-MS) are sensitive and selective. These techniques also have disadvantages: sophisticated tools, high maintenance costs. Therefore, it is necessary to develop a sensory, sensitive, selective, portable platform for heavy metals [1].

It has recently been shown that electrochemical techniques such as differential pulse voltammetry (DPV) and square wave anodic stripping voltammetry (SWASV) using modified electrodes are alternative and very attractive methods for detecting heavy metals [2].

Carbon nanotubes (CNTs) become materials used to modify electrodes due to their unique properties, such as the increased surface area of the electrode, rapid electron transfer, significant mechanical strength and good chemical stability. Recent studies have shown that CNTs provide strong electro-

*email: mpopescu@imnr.ro 
catalytic activity [3]. CNT-modified electrodes promote electron transfer due to their conductivity and mechanical properties.

Also, conductive polymers such as polyaniline [4,5] polyacetylene, polypyrrole, polythiophene, polyphenols have been extensively studied due to their unique physical, chemical, and electrical properties: good processability, environmental stability, low cost, easily synthesized, charge transfer ability, adaptation of properties by doping, etc. [1]. Among these, PANI is one of the most important polymers, due to its stability and electrical conductivity. Moreover, it exhibits significant redox behavior. The conductive form of polyaniline is protonated poly-emeraldine or green poly-emeraldine salt, with a conductivity of about $15 \mathrm{~S}^{*} \mathrm{~cm}^{-1}$. Composites based on conductive polymers and CNT have synergistic effects and have been studied for various applications such as lithium-ion batteries, supercapacitors, catalysts, solar cells, nanodevices, chemical sensors and biosensors [6,7]. Numerous researchers have also used the beneficial, unique and attractive properties resulting from the combination of conductive polymers with CNTs for the detection of heavy metals [1]. The formation of conductive polymer-CNT composites takes place through $\pi-\pi$ interactions between the two components, which favors the improvement of electrochemical performance. Z. Wang et al [8] studied the performance of the glassy carbon electrode (GCE) coated with a nanocomposite based on multi-walled carbon nanotubes (MWCNT) and PANI to be used as an electrochemical sensor for the determination of Pb (II) ions.

In the present study, CNT-PANI composites were prepared by the hydrothermal method in mild synthesis conditions (aqueous solution, temperature $<100^{\circ} \mathrm{C}$ and moderate pressure $\sim 60 \mathrm{~atm}$ ), as previously reported by us in [9] and tested to obtain CNT-PANI thin films on glassy carbon electrode (GCE) by electrochemical methods such as cyclic voltammetry (CV) and differential pulse voltammetry (DPV). The aim of this paper is to demonstrate the potential of hydrothermal process combined with electrochemical techniques to obtain modified electrodes based on functionalized carbon nanotubes (CNT) and electroactive polymers such as Polyaniline (PANI) for metals detection. For this purpose, commercial nanotubes (MWCNT) were functionalized by a mixture of $\mathrm{HNO}_{3} / \mathrm{H}_{2} \mathrm{SO}_{4}$ and further used for hydrothermal synthesis of CNT-PANI composites with different mass ratios. The resulted powders were dispersed in a surfactant/electrolyte solution for preliminary electrochemical experiments (CV and DPV) meant to lead to modified electrodes.

\section{Materials and methods}

\subsection{Materials}

Multi-walled carbon nanotubes (MWCNT) having outer diameter of $10 \mathrm{~nm}$, inner diameter of 4.5 $\mathrm{nm}$, and length of $4 \mu \mathrm{m}$ were purchased from Sigma Aldrich; commercial Polyaniline (emeraldine base, $\mathrm{Mw} \sim 10,000)$ - PANI (Sigma Aldrich) has been used. Sodium hydroxide $(\mathrm{NaOH})$ in solid form was also purchased from Sigma Aldrich. 95-97\%; sulfuric acid is Merck reagent, 65\% nitric acid and 35\% hydrochloric acid are from LACH-NER. The solutions used for titration, $0.05 \mathrm{M} \mathrm{NaOH}$ and $0.05 \mathrm{M} \mathrm{HCl}$, were prepared from solid sodium hydroxide and concentrated hydrochloric acid. Sodium dodecylbenzenesulfonate (SDBS), from Merck, was used as a surfactant as a $2 \mathrm{M}$ aqueous solution. Potassium ferrocyanide $\mathrm{K}_{4}\left[\mathrm{Fe}(\mathrm{CN})_{6}\right] * 3 \mathrm{H}_{2} \mathrm{O}$ (from Merck) was used in the electrochemical experiments as a $0.1 \mathrm{M}$ aqueous solution.

\subsection{CNT functionalization}

MWCNTs were purified by heat treatment in a CARBOLITE oven for $2 \mathrm{~h}$ at a temperature of $400^{\circ} \mathrm{C}$ with a heating rate of $10^{\circ} \mathrm{C} / \mathrm{min}$ and cooling to room temperature. Chemical oxidation was performed using a mixture of nitric acid and sulfuric acid. To avoid destruction of the nanotubes, the solutions of nitric acid and sulfuric acid used for the oxidation of the nanotubes had the following concentrations: 4.0 $\mathrm{M}$ and 10.0 M, respectively. Functionalization of MWCNTs was performed according to the following method: heat-treated MWCNT powder was introduced into a mixture of $4.0 \mathrm{M} \mathrm{HNO}_{3}$ and $10.0 \mathrm{M} \mathrm{H}_{2} \mathrm{SO}_{4}$ (1: 3 volume ratio). The solution was magnetically stirred for $8 \mathrm{~h}$ at room temperature. The resulting suspension was washed with distilled water under magnetic stirring, followed by 
sedimentation and settling. The resulted sediment was further subjected to successive washes (in the same way: stirring, sedimentation, settling) until the $p \mathrm{H}$ of the suspension was $\sim 5.5$. Subsequently, the CNT slurry was freeze-dried in Martin Christ Alpha 1-2 LD Plus equipment.

\subsection{Determination of the degree of functionalization of CNT by acid-base titration}

The titration method proposed by Boehm is used to determine the relative concentration of functional groups on the MWCNT surface. In a typical experiment, $2 \mathrm{mg}$ of oxidized MWCNT are immersed in 20 $\mathrm{mL}$ of $0.05 \mathrm{M} \mathrm{NaOH}$ solution. The mixture is ultrasonicated for $5 \mathrm{~min}$ in a closed flask and then stirred continuously for $24 \mathrm{~h}$ to reach equilibrium. To determine the excess of $\mathrm{NaOH}$ after the acid-base reaction of the MWCNT functionalized with the $\mathrm{NaOH}$ solution, the solution is slowly titrated with $0.05 \mathrm{M} \mathrm{HCl}$ to neutral $p \mathrm{H}$. The concentration of acidic groups on the surface of MWCNT (mmol / gram of MWCNT) is equal to the amount of reacted $\mathrm{NaOH}[10]$.

\subsection{Hydrothermal synthesis of composites based on functionalized CNT and PANI}

Functionalized CNT prepared as described above, was dispersed in water and mixed under magnetic stirring with commercial PANI. The mixture was then brought to neutral $p \mathrm{H}$ with $0.1 \mathrm{M} \mathrm{NaOH}$ and placed in the synthesis vessel. The composite powders with different mass ratios based on functionalized MWCNT and PANI were obtained by the hydrothermal process at 60 atm and $40^{\circ} \mathrm{C}$ (Table 1). The products resulting from the synthesis were freeze-dried at $-50^{\circ} \mathrm{C}$ and $0.025 \mathrm{mbar}$.

\subsection{Structural and thermal characterization of functionalized CNT, respectively CNT-PANI}

Structural analysis by spectral methods: The presence of functional groups was identified by Fourier transform infrared spectroscopy (FT-IR), using an ABB MB 3000 FT-IR spectrometer (Canada), equipped with EasiDiff device for powders analysis. The solid sample (powders presented in Table 1) is mixed with $\mathrm{KBr}$ so that its concentration in the mixture is $1 \%$ gravimetric. The mixture thus obtained is ground for 10-15 min to obtain fine, homogeneous particles. For data acquisition, 64 scans were made at a resolution of $4 \mathrm{~cm}^{-1}$ in the range of $550-4000 \mathrm{~cm}^{-1}$ in transmittance mode. Experimental data processing was performed using Horizon $\mathrm{MB}^{\mathrm{TM}}$ FT-IR software.

Thermal analysis: The study of the thermal transformations due to the chemical reactions was performed by differential scanning calorimetry (DSC) using a NETZSCH DSC 200 F3 differential scanning calorimeter in Ar atmosphere. One has worked in $\mathrm{Al}$ crucibles up to a maximum temperature of $590^{\circ} \mathrm{C}$, with a heating rate of $10 \mathrm{~K} / \mathrm{min}$ and cooling rate of $30 \mathrm{~K} / \mathrm{min}$, respectively. The processing of the experimental data was performed with the help of the PROTEUS ANALYSIS software.

\subsection{Preliminary electrochemical characterization of CNT-PANI}

The electrochemical measurements of the stable suspensions based on CNT, respectively CNT-PANI were performed with a PGSTAT 12 Autolab potentiostat (Methrom) at room temperature. The potentiostat is connected to a computer endowed with NOVA 2.1 software for selecting parameters and collecting data of the electrochemical methods used (CV, DPV).

The electrochemical cell used was a conventional cell with three electrodes: a working electrode glassy carbon disk (diameter $3 \mathrm{~mm}$ ), a reference electrode $\mathrm{Ag} / \mathrm{AgCl}$ in saturated $3 \mathrm{M} \mathrm{KCl}$ solution and a platinum counter-electrode. The working electrode (GCE) was cleaned by sanding on felt with diamond paste $(0.25 \mu \mathrm{m})$ after each recording.

The electrochemical experiments were performed according to the following protocol: 1) cyclic voltammetry in potassium ferrocyanide solution $\mathrm{K}_{4}\left[\mathrm{Fe}(\mathrm{CN})_{6}\right] 0.1 \mathrm{M}$, scan rate of $100 \mathrm{mV} / \mathrm{s} ; \varepsilon 0=-0.3 \mathrm{~V}$; $\varepsilon 1=+0.7 \mathrm{~V} ; \varepsilon 2=-0.3 \mathrm{~V}$ (preliminary experiment for the study of the redox behavior of $\mathrm{K}_{4}\left[\mathrm{Fe}(\mathrm{CN})_{6}\right]$ in the presence of unmodified GCE; 2) cyclic voltammetry with a scan rate of $0.1 \mathrm{~V} / \mathrm{s} ; \varepsilon 0=0 ; \varepsilon 1=+3 \mathrm{~V}$; $\varepsilon 2=-3 \mathrm{~V}$ in $0.2 \mathrm{M}$ SDBS surfactant / electrolyte support solution; 3) cyclic voltammetry with a scan rate of $0.1 \mathrm{~V} / \mathrm{s} ; \varepsilon 0=0 ; \varepsilon 1=+3 \mathrm{~V} ; \varepsilon 2=-3 \mathrm{~V}$ in functionalized carbon nanotube suspension (NT-1), in the presence of SDBS; 4) differential pulse voltammetry with a scan rate of $0.01 \mathrm{~V} / \mathrm{s}$ with a modulation 
amplitude of $0.025 \mathrm{~V}$ and a modulation time of $0.5 \mathrm{~s}$ in the anodic direction (from $0 \mathrm{~V}$ to $3 \mathrm{~V}$ ) and then in the cathodic direction (from $0 \mathrm{~V}$ to $-3 \mathrm{~V}$ ) in functionalized carbon nanotube suspension (NT-1), in the presence of SDBS; 5) cyclic voltammetry with a scan rate of $0.1 \mathrm{~V} / \mathrm{s} ; \varepsilon 0=0 ; \varepsilon 1=+3 \mathrm{~V} ; \varepsilon 2=-3 \mathrm{~V} ; 20$ scans in functionalized carbon nanotube suspension, in the presence of SDBS; 6) transfer of the GC electrode modified with functionalized carbon nanotubes in ferrocyanide solution for cyclic voltammetry, scan rate of $0.1 \mathrm{~V} / \mathrm{s} ; \varepsilon 0=-0.3 \mathrm{~V} ; \varepsilon 1=+0.7 \mathrm{~V} ; \varepsilon 2=-0.3 \mathrm{~V}$. Similarly, steps $3-6$ were repeated for CNTPANI composites (stable suspensions of NTP-1 to NTP-4 powder samples, (Table 1)).

\section{Results and discussions}

The investigated samples, based on functionalized CNT and PANI are presented in the table below:

Table 1. Powdered materials based on functionalized CNT, respectively functionalized CNT and PANI

\begin{tabular}{|c|c|c|c|c|c|}
\hline $\begin{array}{c}\text { Crt. } \\
\text { no }\end{array}$ & Sample name & $\begin{array}{c}\text { CNT : PANI } \\
\text { mass ratio }\end{array}$ & Synthesis time, h & pH & Sample type \\
\hline 1 & NT-1 & - & - & 5.5 & Chemically oxidized MWCNT \\
\hline 2 & NT-2 & - & - & 5.5 & Chemically oxidized MWCNT \\
\hline 3 & NTP-1 & $1: 2$ & 3 & 7 & $\begin{array}{c}\text { Hydrothermally synthesized CNT-PANI at } \\
40^{\circ} \mathrm{C} / 60 \text { atm }\end{array}$ \\
\hline 4 & NTP-2 & $1: 4$ & 3 & 7 & $\begin{array}{c}\text { Hydrothermally synthesized CNT-PANI at } \\
40^{\circ} \mathrm{C} / 60 \text { atm }\end{array}$ \\
\hline 5 & NTP-3 & $1: 2$ & 1 & 7 & $\begin{array}{c}\text { Hydrothermally synthesized CNT-PANI at } \\
40^{\circ} \mathrm{C} / 60 \text { atm }\end{array}$ \\
\hline 6 & NTP-4 & $1: 4$ & 1 & $\begin{array}{c}\text { Hydrothermally synthesized CNT-PANI at } \\
40^{\circ} \mathrm{C} / 60 \text { atm }\end{array}$ \\
\hline
\end{tabular}

\subsection{Structural analysis by spectral methods (FT-IR)}

The influence of the reaction parameters on the formation of composite structures based on MWCNT and PANI was studied using Fourier-transform Infrared Spectroscopy (FT-IR). Pure PANI and functionalized CNT were also analyzed for comparative reasons. Thus, Figure 1a shows the FT-IR spectra of the functionalized MWCNT samples $(p \mathrm{H}=5.5)$ and Figure $1 \mathrm{~b}$ - the FT-IR spectra of the commercial PANI. In the Figure 2, the FT-IR spectra of the composite materials based on MWCNT and PANI (polyaniline) are presented.

The FT-IR spectra of chemically oxidized CNTs show two absorption maxima, barely visible, at $1597-1550 \mathrm{~cm}^{-1}$ and $1686-1650 \mathrm{~cm}^{-1}$, attributed to the stretching mode of the $\mathrm{C}=\mathrm{C}$ bond. The number of surface carboxyl groups, calculated based on acid-base titration is $0.97 \mathrm{~g} / \mathrm{mmol}$, which demonstrates a slight functionalization on the CNT surface, in accordance with the results of the spectral analysis.

In the PANI spectrum, the typical vibration bands from $1593 \mathrm{~cm}^{-1}$ and $1508 \mathrm{~cm}^{-1}$ belong to the $\mathrm{C}=$ $\mathrm{C}$ bonds of the quinoid rings, while the maxima from $1499 \mathrm{~cm}^{-1}, 1302 \mathrm{~cm}^{-1}$ and $1242 \mathrm{~cm}^{-1}$ are assigned to $\mathrm{C}=\mathrm{C}$ bonds in benzene rings, $\mathrm{C}-\mathrm{N}$ bond in quinoid rings and $\mathrm{C}-\mathrm{N}$ bond in benzene rings, respectively. Compared to PANI, in the PANI / CNT spectra, two changes can be observed: i) the displacement of the absorption maximum, towards higher wave numbers for the $\mathrm{CN}$ bonds of the quinoid rings (1306-1308 $\mathrm{cm}^{-1}$ ) and ii) the maximum intensity ratio corresponding to the $\mathrm{C}=\mathrm{C}$ bonds in the quinoid ring and the $\mathrm{C}=\mathrm{C}$ bonds of the benzenoid rings increases with the decrease of the ratio between PANI and CNT. The shift to red is due to the $\pi-\pi$ electronic interactions at the interface between PANI and CNT [11]. 

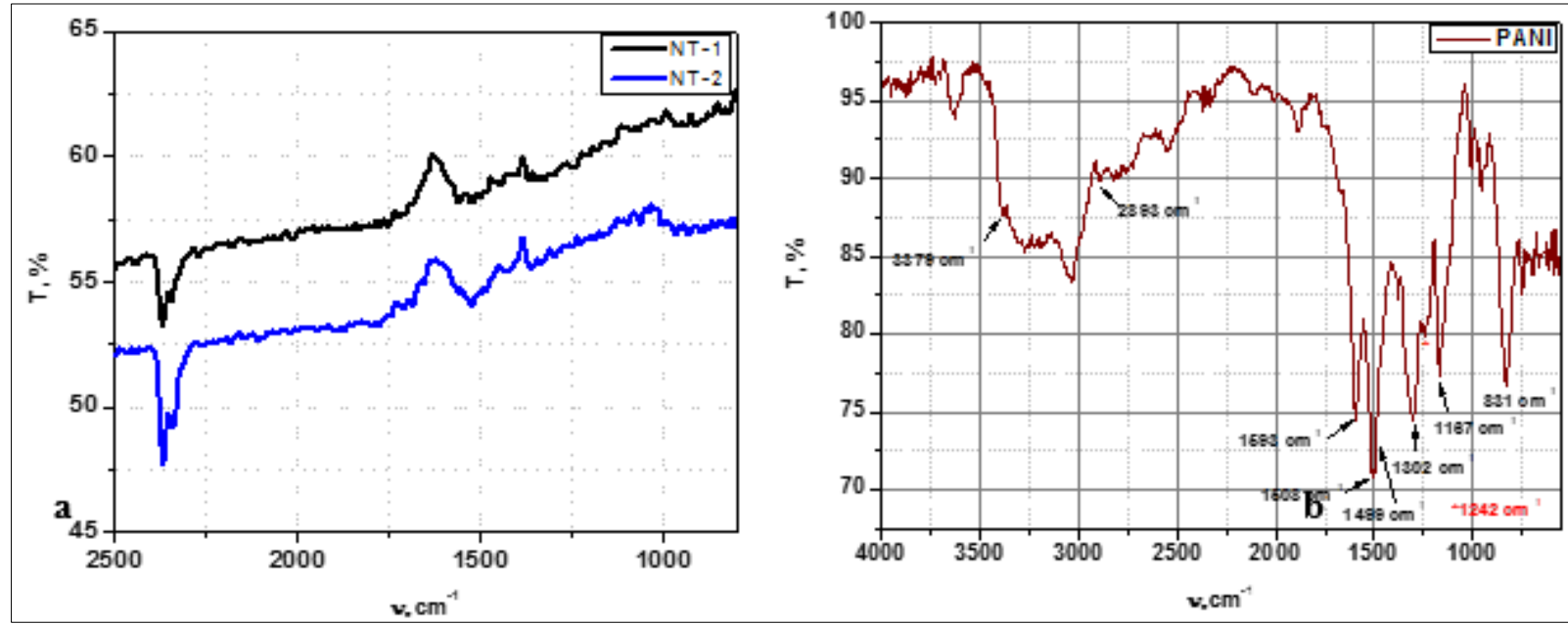

a

b

Figure 1. FT-IR spectra of: a) NT-1 and NT-2 powders

(functionalized CNTs); b) commercial PANI

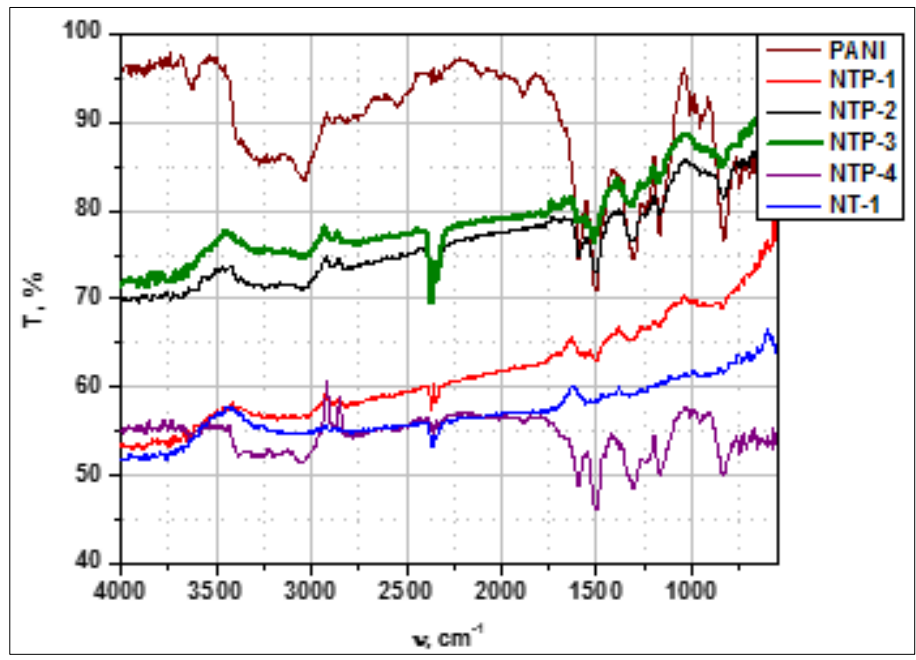

Figure 2. FT-IR spectra of CNT-PANI composite materials, compared to commercial PANI and functionalized CNT (NT-1)

\subsection{Thermal analysis (DSC)}

The results obtained at thermal analysis are presented comparatively in Figure $3(a, b, c)$ and Table 2. As observed in Figure 3a, the functionalized CNT sample (NT-1) decomposes into $\mathrm{CO}_{2}$ at $435.5{ }^{\circ} \mathrm{C}$, which proves the functionalization of $\mathrm{CNT}$ by the presence of carboxyl groups $(\mathrm{COOH})$ on the surface. In the case of polyaniline (Figure 3d), the peak corresponding to the water loss or unreacted monomer is observed at $92.2^{\circ} \mathrm{C}$, while the decomposition peak of the organic phase can be observed at $316.6^{\circ} \mathrm{C}$.

In the case of composite samples (Figure 3b) with CNT-PANI mass ratio=1:2 (NTP-1 and 3) the decomposition of the organic phase can be observed at $368.6^{\circ} \mathrm{C}$ and $352.5^{\circ} \mathrm{C}$, respectively. The endothermic peak from $443-489.9^{\circ} \mathrm{C}$, due to carbon nanotubes can be also found, but the enthalpy (peak area) is very small in the case of NTP-1. It should be noted that in the case of the NTP-2 sample (Figure $3 \mathrm{c}$ ), synthesized for $3 \mathrm{~h}, \mathrm{CNT}$ : PANI mass ratio $=1: 4$, the peak due to the loss of the organic phase disappears. This aspect could be explained by a better interaction of CNT with PANI, due to the fact that the hydrothermal reactions are diffusion-controlled processes [12]. Peak area corresponding to the decomposition of the organic phase is small in the case of NTP-4 sample (CNT: PANI mass ratio=1:4, synthesis time $=1 \mathrm{~h}$ ). NTP-3 sample (Figure $3 \mathrm{~b}$ ), synthesized for $1 \mathrm{~h}$, with CNT: PANI mass ratio $=1: 2$ has the highest enthalpy (peak area) due to PANI decomposition, confirming the hypothesis that a longer synthesis time (3h) and a higher organic phase content leads to a better interaction of PANI with CNT. 
Peaks observed between 165 and $169^{\circ} \mathrm{C}$ in all the composite powders correspond to a transition in PANI (endothermic affect at $177^{\circ} \mathrm{C}$ ) which could be attributed to some changes in polymer chain [13].

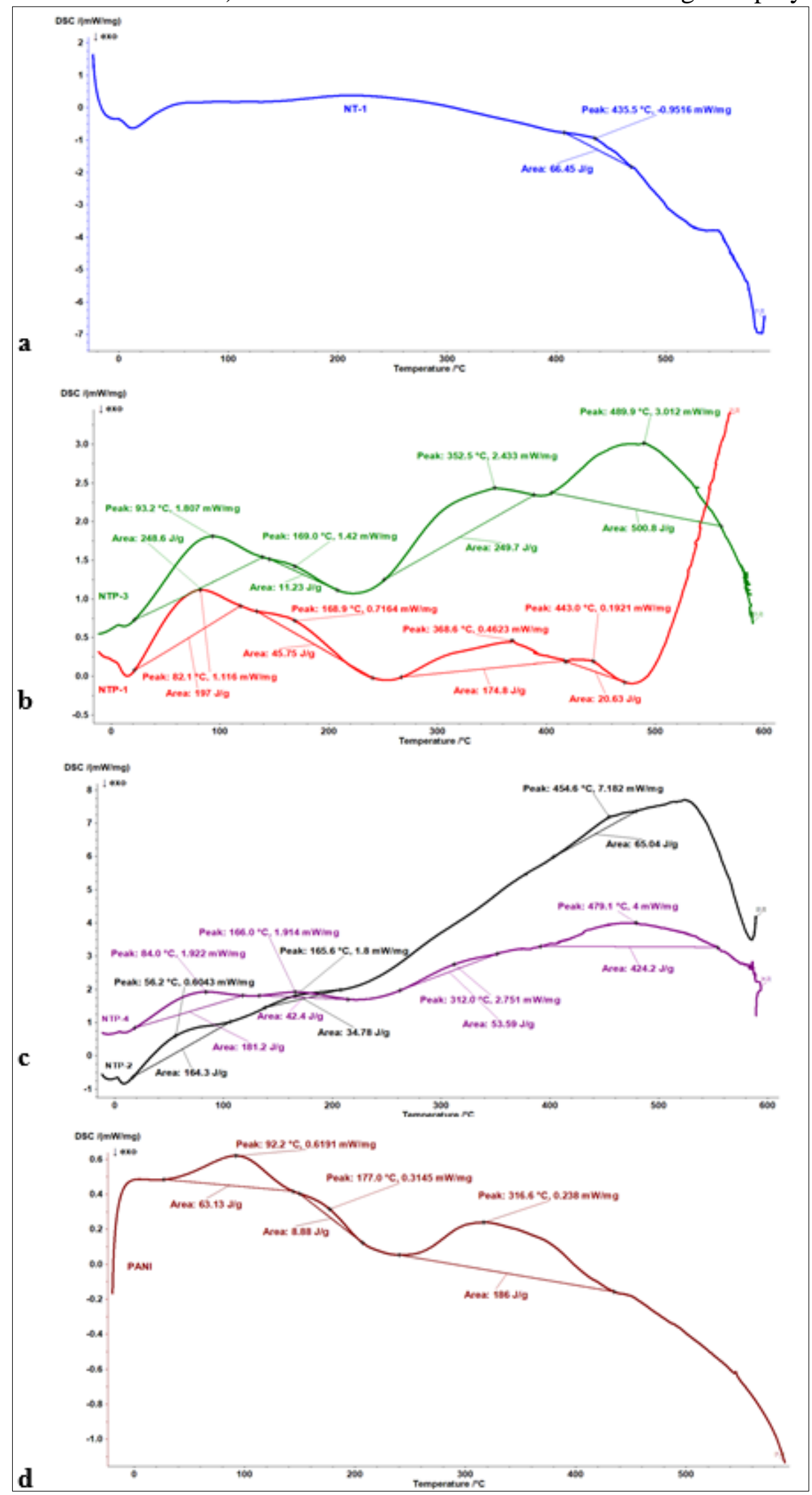

Figure 3. DSC curves of a) NT-1 powder; b) NTP-1 and NTP-3 powders;

c) NTP-2 and NTP-4 powders; d) commercial PANI 
Table 2. Peak temperatures and enthalpies of functionalized CNT, CNT-PANI composites and PANI

\begin{tabular}{|c|c|c|c|c|c|c|c|c|c|c|}
\hline \multirow[b]{2}{*}{$\begin{array}{c}\text { Crt. } \\
\text { no }\end{array}$} & \multirow[b]{2}{*}{$\begin{array}{c}\text { Sample } \\
\text { name }\end{array}$} & \multirow{2}{*}{$\begin{array}{c}\text { CNT : PANI } \\
\text { mass ratio }\end{array}$} & \multicolumn{2}{|c|}{ Peak 1} & \multicolumn{2}{|c|}{ Peak 2} & \multicolumn{2}{|c|}{ Peak 3} & \multicolumn{2}{|c|}{ Peak 4} \\
\hline & & & $\mathbf{T}_{1},{ }^{\circ} \mathbf{C}$ & $\Delta \mathbf{H}_{1}, \mathrm{~J} / \mathrm{g}$ & $\mathbf{T}_{2},{ }^{\circ} \mathbf{C}$ & $\begin{array}{c}\Delta \mathbf{H}_{2}, \\
\mathbf{J} / \mathrm{g}\end{array}$ & $\mathbf{T}_{3},{ }^{\circ} \mathbf{C}$ & $\begin{array}{c}\Delta \mathbf{H}_{3}, \\
\mathbf{J} / g\end{array}$ & $\mathbf{T}_{\mathbf{4}},{ }^{\circ} \mathbf{C}$ & $\Delta \mathbf{H}_{4}, \mathrm{~J} / \mathrm{g}$ \\
\hline 1 & NT-1 & - & - & - & & & - & - & 435.5 & 66.45 \\
\hline 2 & NTP-1 & $1: 2$ & 82.1 & 197 & 168.9 & 45.75 & 368.6 & 174.8 & 443 & 20.63 \\
\hline 3 & NTP-2 & $1: 4$ & 56.2 & 164.3 & 165.6 & 34.78 & - & - & 454.6 & 65.04 \\
\hline 4 & NTP-3 & $1: 2$ & 93.2 & 248.6 & 169 & 11.23 & 352.5 & 249.7 & 489.9 & 500.8 \\
\hline 5 & NTP-4 & $1: 4$ & 84 & 181.2 & 166 & 42.4 & 312 & 53.59 & 479.1 & 424.2 \\
\hline 6 & PANI & - & 92.2 & 63.13 & 177 & 8.88 & 316.6 & 186 & - & - \\
\hline
\end{tabular}

Results obtained by FT-IR and DSC analysis of CNT-PANI composite powders shows that NTP-2 sample synthesized for $3 \mathrm{~h}$, with CNT:PANI mass ratio $=1: 4$ has the best structural and thermal characteristics among all the composite materials investigated in this study.

\subsection{Preliminary electrochemical tests}

The electrochemical methods used for the preliminary characterization of hybrid materials based on CNT functionalized with PANI are: cyclic voltammetry $(\mathrm{CV})$ with scan rate $0.1 \mathrm{~V} / \mathrm{s}$ and differential pulse voltammetry (DPV) with scan rate of $0.01 \mathrm{~V} / \mathrm{s}$. Peaks were obtained both in the anodic and cathodic domain. The anodic and cathodic curves were recorded individually, starting from the stationary potential. Cyclic voltammetry $(\mathrm{CV})$ is a dynamic electrochemical measurement where the potential is ramped linearly versus time. The responses are anodic and/or cathodic peak currents that are proportional to the concentration of the electroactive species. This technique is used for electrochemical characterization of working electrodes. Differential pulse voltammetry (DPV) is one of the main pulsed techniques used in biosensing. The main advantage exhibited by this technique is the low capacitive current, which can improve the sensitivity of the voltammetric procedures. Differential pulse voltammetry is usually applied in irreversible systems and in systems that present slow-reaction kinetics [14]. Figure 4 shows the CV curves of GC electrode modified with NT1, and NTP1 to NTP4 coatings, respectively. Two oxidation peaks can be observed for the GC electrode modified with NTP2: one located at $\sim 0.6 \mathrm{~V}$ and the other at $\sim 1.8 \mathrm{~V}$. The peak at $0.6 \mathrm{~V}$ could be attributed to the oxidation reaction of emeraldine (conductive form of PANI) in pernigraniline, while the $1.8 \mathrm{~V}$ peak may be due to the slow transfer of electrons between the two forms of PANI (emeraldine-pernigraniline) caused by the steric hindrance of the polymer chain (Figure 5) $[15,16]$.

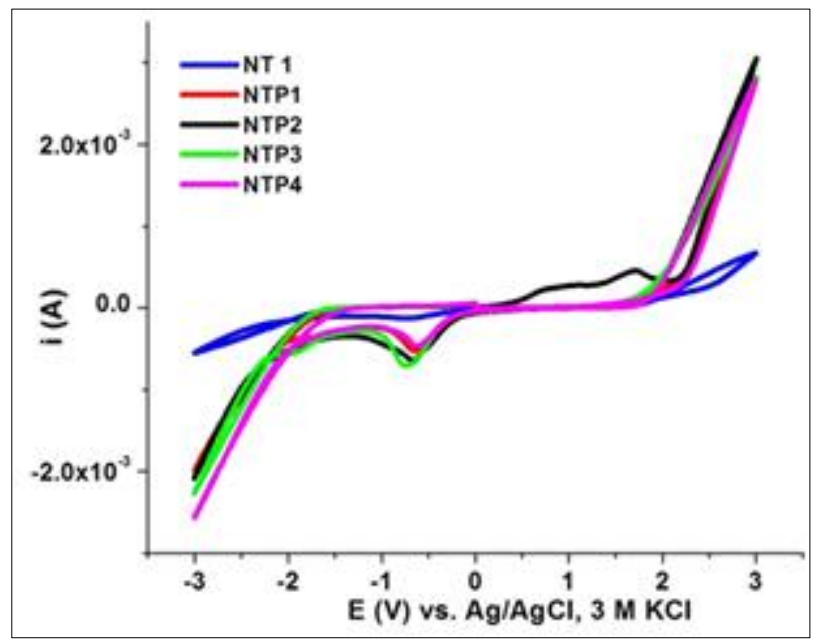

Figure 4. Cyclic

voltammograms $(\mathrm{CV})$ of GCE modified with the studied coatings (denoted as NT1, NTP1, NTP2, NTP3 and

NTP4), in the potential range $-3 \mathrm{~V} \div 3 \mathrm{~V}$ 


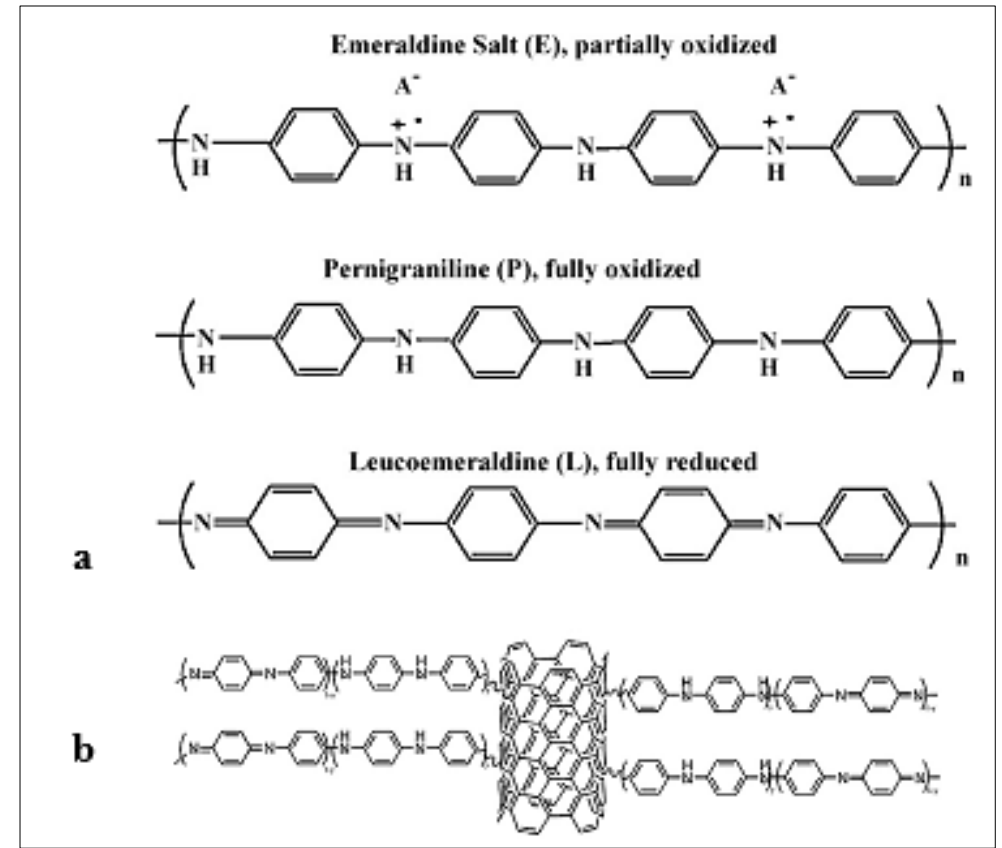

Figure 5. a) the oxidation states of PANI [17]; b) example of CNT-PANI structure [11]

In the reduction range a single peak at $\sim-0.7 \mathrm{~V}$ is observed for all the modified electrodes. This peak could be justified by the presence of carbon nanotubes that increase PANI electroactivity by charge transfer reactions [17]. It is also known that chemically synthesized PANI has a reversible peak $(\varepsilon$ cathodic $=-0.7 \mathrm{~V}$ corresponds to $\varepsilon$ anodic $=1.8 \mathrm{~V}$ ) [18]. The results obtained by CV for GCE modified with NTP2 coating correlates well with the conclusions on NTP-2 composite powder, confirming the presence of the organic phase on GC modified electrode.

Figure 6 shows the DPV curves of GC electrode modified with NT1, and NTP1 to NTP4 coatings, respectively. For electrodes modified with composite coatings (NTP1 to NTP4), an oxidation peak is observed at the potential of $\sim 1.4 \mathrm{~V}$, probably due to the oxidation reaction of emeraldine to pernigraniline, in accordance with the results obtained at cyclic voltammetry. In the reduction range all the GC modified electrodes have a main reduction peak at $-0.6 \mathrm{~V}$. The peak at $-0.6 \mathrm{~V}$ is shifted from cyclic voltammetry $(-0.7 \mathrm{~V})$ because in the case of DPV the scan rate is lower, DPV being a more precise method, in which the Faradaic currents are better discriminated (electron transfer on and from the electrode).

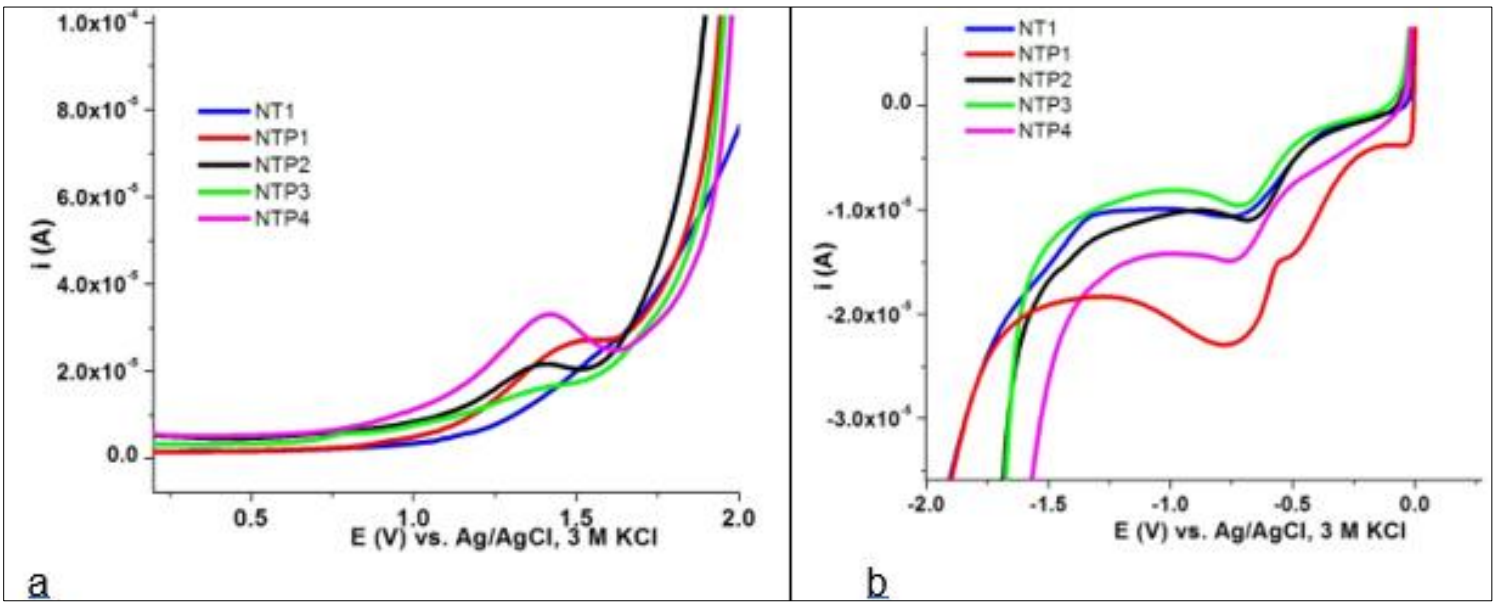

Figure 6. Differential pulse voltammetry (DPV) curves of glassy carbon electrode modified with the studied coatings in the: a) anodic range and b) cathodic range 
Figure 7 shows the $\mathrm{CV}$ curves of 20 successive cycles for GC electrode modified with NT1, and NTP1 to NTP4 coatings, respectively. The decrease of the current with the increase of the number of cycles can be observed. The transfer of the electrodes after their modification by successive cycles (Figure 8) was carried out in a solution of potassium ferrocyanide $\left(\mathrm{K}_{4}\left[\mathrm{Fe}(\mathrm{CN})_{6}\right]\right.$ (cyan curve). It can be observed that the signal of the electrode modified by successive cycles with NTP1 and NTP3 coatings, respectively, is changed compared to the bare glassy carbon electrode. The signal of the electrode modified with NT1 coating shows a thinner film compared to the electrode modified with NTP1 and NTP3 coatings.

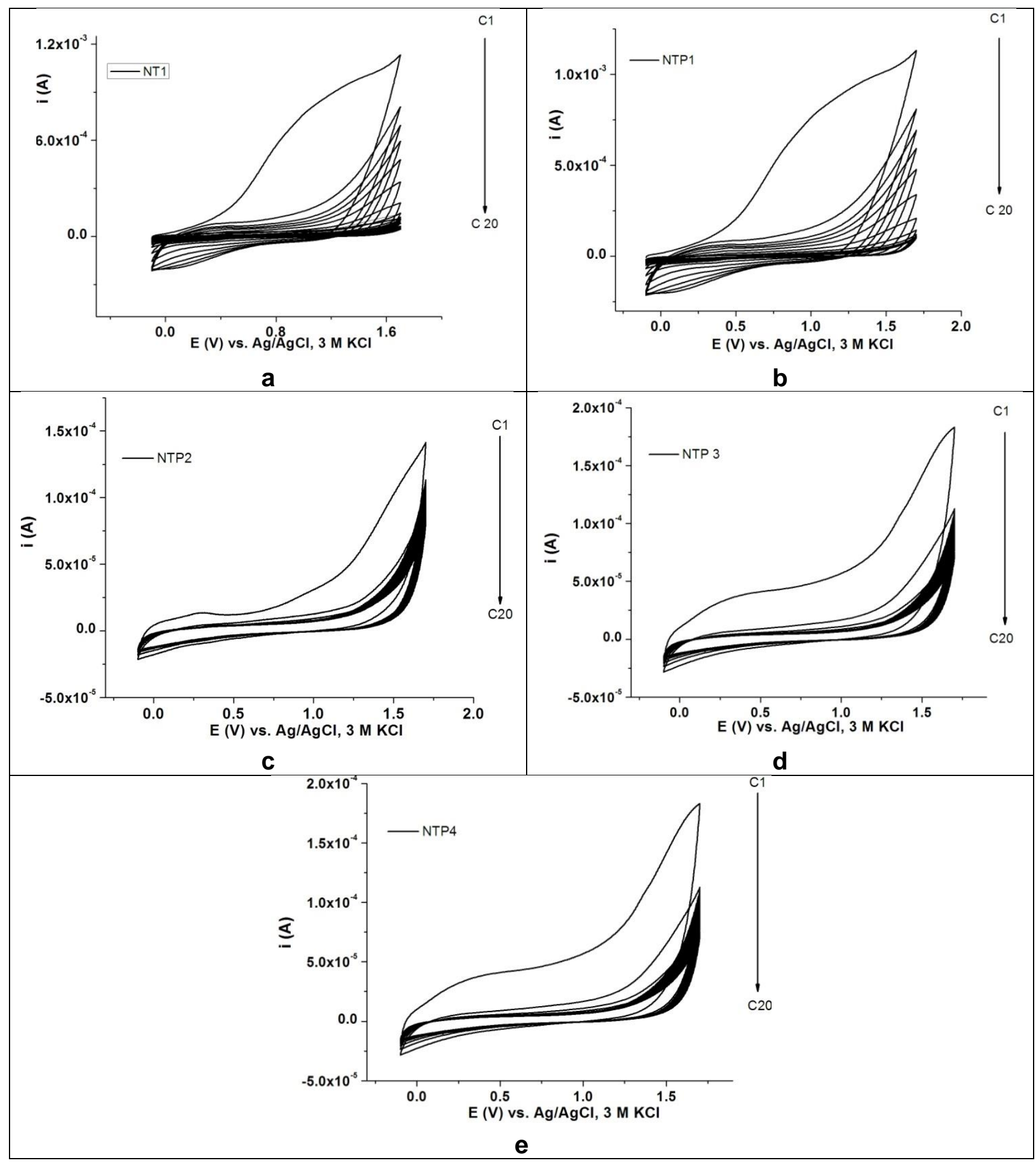

Figure 7. Cyclic voltammetry curves of glassy carbon electrode (GC) modified with the studied coatings, in the potential range $-3 \mathrm{~V} \div 3 \mathrm{~V}, 20$ cycles 
The electrodes modified with NTP2 and NTP4 coatings have currents with the lowest intensity, which indicates a poorly conductive film. It has been observed that, as the anodic potential limit is more positive, the potassium ferrocyanide signal is more flattened and lower intensity currents are obtained, which indicates the formation of an insulating film.

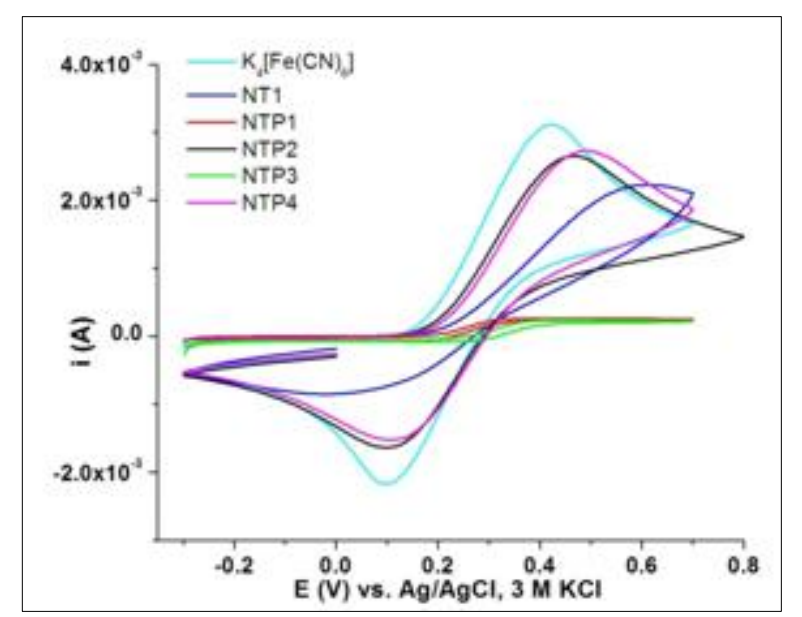

Figure 8. Cyclic voltammograms of the potassium ferrocyanide solution at bare GCE (cyan curve) and modified with the studied coatings

\section{Conclusions}

In this paper, composite materials based on functionalized carbon nanotubes and polyaniline were synthesized, in order to obtain glassy carbon electrode modified with CNT - polymer coatings. The influence of the CNT: PANI mass ratio and the synthesis time on the formation of composites with the desired structural and electrochemical properties were studied. It was found that the NTP-2 powder, with CNT: PANI mass ratio 1: 4 , and $3 \mathrm{~h}$ synthesis time, represents the best choice, having the best structural and thermal characteristics and forming a weakly conductive film on the surface of the glassy carbon electrode. Preliminary electrochemical tests revealed the electroactive forms of polyaniline, through the presence of characteristic oxidation peaks but also reduction peaks, corresponding to reversible redox reactions, demonstrating that glassy carbon electrode has been modified by electrochemical methods (CV and DPV) with coatings of CNT-PANI.

The results obtained by $\mathrm{CV}$ for GCE modified with NTP2 coating correlates well with the conclusions on NTP-2 composite powder, confirming the presence of the organic phase on GC modified electrode.

Further studies will be conducted to test the potential application of glassy carbon electrode modified with CNT-PANI thin films as electrochemical sensor for heavy metals detection.

Acknowledgments: This work was performed through the Core Program, carried out with the support of MCI, project no. PN19190501/2019-2022, "Innovative electrochemical processes with applications in surface engineering and non-ferrous metal recovery". The authors thank to dr. eng. Marian-Laurentiu Tatu for electrochemical measurements and fruitful discussions on electrochemistry.

\section{References}

1.Deshmukh, M. A., Shirsat, M. D., Ramanaviciene, A., Ramanavicius, A. Composites Based on Conducting Polymers and Carbon Nanomaterials for Heavy Metal Ion Sensing (Review). Crit. Rev. Anal. Chem. 48, 2018, 293-304.

2.WAHEED, A., MANShA, M., Ullah, N. Nanomaterials-based electrochemical detection of heavy metals in water: Current status, challenges and future direction. TrAC - Trends Anal. Chem. 105, 2018, 37-51. 
3.XIE, F. ET AL. Carbon-based nanomaterials - A promising electrochemical sensor toward persistent toxic substance. TrAC - Trends Anal. Chem. 119, 2019, 115624.

4.Bhadra, S., Khastgir, D., Singha, N. K., LeE, J. H. Progress in preparation, processing and applications of polyaniline. Prog. Polym. Sci. 34, 2009, 783-810.

5.MAndić, Z., Roković, M. K., POKUPČIĆ, T. Polyaniline as cathodic material for electrochemical energy sources. The role of morphology. Electrochim. Acta 54, 2009, 2941-2950.

6.SABZI, R. E., REZAPOUR, K., SAMADI, N. Polyaniline-multi-wall-carbon nanotube nanocomposites as a dopamine sensor. J. Serbian Chem. Soc. 75, 2010, 537-549.

7.NGUYEN, V. H., SHIM, J. J. Green synthesis and characterization of carbon nanotubes/polyaniline nanocomposites. J. Spectrosc. 2015, 2015,.

8.WANG, Z., LIU, E., GU, D., WANG, Y. Glassy carbon electrode coated with polyaniline-functionalized carbon nanotubes for detection of trace lead in acetate solution. Thin Solid Films 519, 2011, 5280-5284. 9.Cursaru Popescu, L. M., Plaiasu, A. G., Ducu, C. M., Piticescu, R. M., Tudor, I. A. Carbon Nanotube/Polyaniline Composite Films Prepared by Hydrothermal- Electrochemical Method for Biosensor Applications. Proc. Int. Semicond. Conf. CAS 2018-Octob, 2018, 249-252.

10.THI MAI HOA, L. Characterization of multi-walled carbon nanotubes functionalized by a mixture of HNO3/H2SO4. Diam. Relat. Mater. 89, 2018, 43-51.

11.RUI, M., JiANG, Y., ZHU, A. Sub-micron calcium carbonate as a template for the preparation of dendrite-like PANI/CNT nanocomposites and its corrosion protection properties. Chem. Eng. J. 385, 2020 ,

12.Piticescu, R. M., Piticescu, R. R., Taloi, D., Badilita, V. Hydrothermal synthesis of ceramic nanomaterials for functional applications. Nanotechnology 14, 2003, 312-317.

13.Kumar, D., Chandra, R. Thermal behaviour of synthetic metals: Polyanilines. Indian J. Eng. Mater. Sci. 8, 2001, 209-214.

14.BraZaCa, L. C., Ribovski, L., Janegitz, B. C., Zucolotto, V. Nanostructured materials and nanoparticles for point of care (POC) medical biosensors. Medical Biosensors for Point of Care (POC) Applications vol. 7 (Elsevier Ltd, 2017).

15.MAteos, M. ET AL. Comprehensive Study of Poly(2,3,5,6-tetrafluoroaniline): From Electrosynthesis to Heterojunctions and Ammonia Sensing. ACS Appl. Mater. Interfaces 10, 2018, 19974-19986.

16.Mondal, S., Sangaranarayanan, M. V. Permselectivity and thickness-dependent ion transport properties of overoxidized polyaniline: A mechanistic investigation. Phys. Chem. Chem. Phys. 18, 2016, 30705-30720.

17.Jamadade, V. S., Dhawale, D. S., LokHande, C. D. Studies on electrosynthesized leucoemeraldine, emeraldine and pernigraniline forms of polyaniline films and their supercapacitive behavior. Synth. Met. 160, 2010, 955-960.

18.SHABIR HuSSEIn SomjeE RAJAN, Z. Electrochemical Characterisation Methodology. 2019, 1-6.

$\overline{\text { Manuscript received: 09.09.2020 }}$ 